\title{
Young's modulus estimation in food samples: effect of experimental parameters
}

\author{
Ankita Sinha and Atul Bhargav* \\ Energy Systems Research Laboratory, Mechanical Engineering Discipline, Indian Institute of Technology Gandhinagar, Palaj, \\ Gandhinagar, GJ 382355, India
}

Received: 2 July 2019 / Accepted: 19 March 2020

\begin{abstract}
Texture is an important attribute in the quality assessment of processed food products. Recently, Young's modulus is identified as one of the most important indicators of food texture. However, there is much ambiguity in the literature about quantification and standards for texture analysis. In this paper, the sensitivity of Young's modulus (and thus texture) towards the applied deformation rate, sample shape and size, moisture content is studied experimentally for potato and sweet potato samples. We found that Young's moduli vary by as much as $54 \%$ depending on the rate of applied strain, indicating the need for test standards. The strain rate dependent behaviour exhibits the viscoelastic nature of the potato samples, which was further validated by stress relaxation and cyclic tests. Based on our experimental iterations and associated finding of the work, we propose the need for a standardised procedure for measuring Young's modulus and texture analysis. We expect this work to serve as a crucial step toward standardised texture measurement during thermal processing of food products.
\end{abstract}

Keywords: texture / Young's modulus / food quality / standardization

\section{Introduction}

Texture plays an important role in the acceptability of a food product. This texture is developed throughout the cooking process. Various sensory-based texture characteristics that are identified and analysed so far include firmness, springiness, crispiness, cohesiveness and gumminess [1-8]. Various techniques of texture measurement include texture profile analysis, texture indices measurement and texture modelling [2]. Texture profile analyses involve the development of force-displacement curves that demonstrate the evolution of texture characteristics over time for a food sample. Mechanical and acoustic measurements are used to quantify texture profiles. Mechanical measurements include compression test, three-point bending, puncture test and impact test; these typically yield force vs time and force vs displacement curves. Acoustic measurement techniques involve measurement of acoustic emissions and vibrations associated with texture attributes. Various researchers have incorporated these measurement methods [9-15]. Texture indices are empirical relations derived on the basis of mechanical and/or acoustic measurements. Commonly used texture indices are firmness index [16,17], sharpness index [18] crunchiness index [19] and mastication based texture index [20-22].

\footnotetext{
* e-mail: atul.bhargav@iitgn.ac.in
}

One challenge with these methods is repeatability, owing to the lack of standardization [2]. Besides, while these methods are useful in providing insights about the sensory characteristics of food materials, information regarding quantifiable mechanical properties is very limited. Thus, the utility of these methods is limited while exploring mechanical interpretations of material behaviour. Therefore, mechanical properties and the methods to quantify them have been now extensively studied for various food materials [23-25].

In recent years, Young's Modulus has been identified as one of the key parameters that are used to describe texture $[26,27]$. Unlike other sensory characteristics, Young's modulus is defined on the basis of stress-strain behaviour and not force-time or force-displacement behaviour; which depend on sample dimensions and thus lack generalization. Young's modulus is a direct measure of stiffness, a material property which is crucial in describing the deformation behaviour of materials [28]. Stiffness is defined as the ability of a material to resist deformation under compressive or tensile loading. Researchers have shown that jaw muscle activity is strongly influenced by mechanical properties such as stiffness and the texture behaviour of food samples [29-31]. Reference [32] presented data on Young's modulus of food materials consumed by nonhuman primates and emphasized on the significance of analysing material properties in terms of measurable quantities such as Young's modulus and its role in the 
analysis of masticatory functions. Relationships between Young's modulus and mastication velocity have been established, which demonstrates that higher Young's moduli of food materials result in lower mastication velocity [33]. Similar negative correlations were observed between Young's modulus and mastication velocity through analysis of material properties for four different types of cheeses [34]. The application of knowledge of Young's modulus of food materials in designing their processing machinery has also been studied [35]. Further, they also investigated the effect of loading rate and moisture content on Young's modulus of red beans. Vincent demonstrated the advantages of testing mechanical properties of food materials over sensory experiments and organoleptic tests in terms of convenience, time and cost involved and acceptability [36]. The extensive applicability of Young's modulus and its significance in mechanical studies as mentioned above, justifies the need of its acceptability as a crucial texture parameter. Therefore, Young's modulus has been chosen for quantification of texture in the present work.

A major challenge associated with texture studies involving mechanical parameters such as Young's modulus is the wide variation in estimated values even for very similar samples. These variations demand investigation of the possible causes associated with data disagreements. One explanation is the geographic, seasonal, agricultural and biological reasons for variation. However, it is also possible that some deviations may occur due to the lack of standardised measurement techniques. For instance, the dimensions, aspect ratio, applied strain rate vary significantly across the reported experimental literature $[37,38]$. Thus, there is a need to develop a generalised method for texture analysis of food materials that account for the effect of operating parameters. This could serve as a foundation for the development of standardised testing techniques that could be adopted globally.

The objective of the paper is to study the effect of various physical parameters (geometry, dimensions, aspect ratio, strain rate and moisture content) on Young's modulus. The effect of these parameters on the compressive strength of the food material is also captured. As an outcome of the performed analyses, we also propose the need for a standardised test procedure for Young's modulus and thus texture measurement.

\section{Materials and methods}

Potato (Solanum tuberosum) of Indian variety Kufri Badshah was selected as the primary sample to study the effect of various parameters. In order to further observe the effect of variation in composition and type of sample, additional tests were performed for sweet potato (Ipomoea batatas) samples.

The samples bought from the local market were kept in cold storage before experiments. The samples were brought to room temperature, washed and peeled before cutting. Each measurement of moisture content, Young's modulus and temperature were done in triplicate sets, and the average values were determined. The sample once used for
Table 1. Size and shape details of test cases 1 through 4 (to test for variation in Young's modulus and compressive strength values with size, aspect ratio and geometry).

\begin{tabular}{lllll}
\hline $\begin{array}{l}\text { Test case } \\
\text { Shape }\end{array}$ & 1 & 2 & 3 & 4 \\
\hline $\begin{array}{l}\text { Cross section } \\
\left(\mathrm{mm}^{2}\right)\end{array}$ & $14 \times 14$ & $14 \times 14$ & $7 \times 7$ & 25 (dia) \\
$\begin{array}{l}\text { Height }(\mathrm{mm}) \\
\begin{array}{l}\text { Aspect ratio } \\
(a / l)\end{array}\end{array}$ & 14 & 28 & 14 & 28 \\
\hline
\end{tabular}

measuring a parameter was discarded and was not used for measuring other parameters. Experiments were performed to analyse the effect of sample dimensions, geometry, aspect ratio, applied strain rate and moisture content on Young's modulus of the samples.

For analysing the effect of geometry, cube, cuboid and cylindrical samples were selected for texture analysis. The aspect ratio of $1: 1$ and 1:2 were analysed. Higher aspect ratio were not considered as bulging effect might be significant in those cases. Texture analysis was done for various dimensions of food samples and the effect of these parameters were observed. Details of the selected dimensions, geometry, aspect ratio etc. are shown in Table 1.

The effect of strain rate was analysed by taking raw potato samples cut in the square prismatic shape of $7 \times 7$ $\mathrm{mm}^{2}$ cross-section and height $7 \mathrm{~mm}$ using a mechanical cutter. Strain rate was varied by changing the deformation rate in texture analyser using equation (1), described in the later section of this paper.

For analysing the sensitivity of Young's Modulus and texture towards moisture content, two thermal treatments viz. thermal drying and deep-frying were selected. Potato samples were cut in the square prismatic shape of $14 \times 14$ $\mathrm{mm}^{2}$ cross-section and height $2 \mathrm{~mm}$ using a mechanical cutter. The sample height was kept relatively low to ensure near-uniform heating in the sample. For the drying experiment, samples were dried in the infrared heater at $115^{\circ} \mathrm{C}$ for different levels of moisture loss. For deep frying experiments, samples were fried in corn oil at $170^{\circ} \mathrm{C}$ for different durations ranging from 0 to $7 \mathrm{~min}$. Deep frying was done in a 2-litre electrical fryer (Inalsa Professional2 1700W electric fryer, India). Moisture content was measured by gravimetric analysis done using a preheated convection oven (Nova Instruments, India). Then, compression tests were carried out on samples with different moisture content, starting with fresh samples and ending with samples with less than $10 \%$ moisture content, in steps of $10 \%$ moisture loss. This gives the variation of Young's modulus with moisture content. It should be noted that along with moisture content, starch gelatinization has also an effect on Young's modulus during frying. While no separate experiments were conducted to analyse starch gelatinization, its effect on Young's modulus has been captured and discussed in Section 3.

Texture analyses/compression test: Texture analyses were done using Ta HD plus Texture Analyzer (Stable Microsystems, UK) with a $70 \mathrm{~mm}$ compression platen 


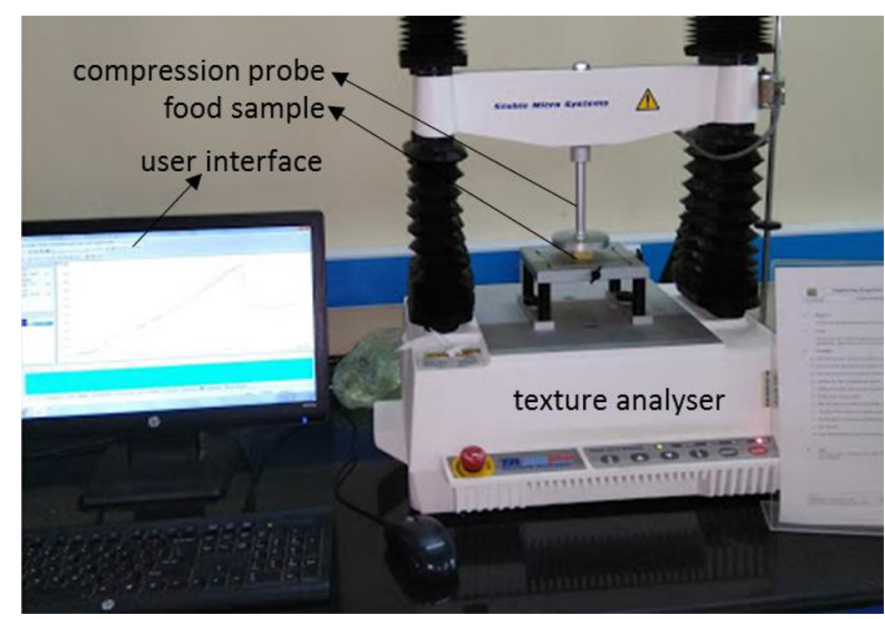

Fig. 1. Texture analysis setup used for compression tests.

probe for applying force (Figure 1). Texture analyser provides the deformation behaviour of food samples under various loading conditions. Displacement rate, defined as the desired change in dimension (here change in sample height) per second is fed as input by the user for texture analysis. However, maintaining the same displacement rate over different experiments is not a correct method as it is dependent on sample dimensions. Instead, a constant strain rate experiment enables comparison across sample sizes $[39,40]$. Therefore, in the current work, a constant strain rate (of $8 \% / \mathrm{min}$ ) is applied for all the cases except the one in which the effect of strain rate is investigated.

Displacement rate was calculated corresponding to desired strain rate by using the following formula:

$$
\begin{aligned}
& \text { strain rate }(\% / \mathrm{min}) \\
& \qquad=\frac{\operatorname{displacement} \text { rate }(\mathrm{mm} / \mathrm{s}) \times 100(\%) \times 60(\mathrm{~s} / \mathrm{min})}{\text { sample height }(\mathrm{mm})}
\end{aligned}
$$

For performing texture analysis, selected food sample was compressed at the specified strain rate till $90 \%$ deformation was achieved. The criteria of $90 \%$ deformation was selected so as to capture the deformation behaviour to the maximum possible extent without damaging the compression platen probe.

Since stress-strain curve is independent of material dimensions, it is a better description of material behaviour vis-à-vis the force-displacement diagram [28]. Therefore stress-strain behaviour was analysed for each sample instead of directly observing force vs displacement. Stress is defined as the force per unit area and was calculated by dividing force values with the sample cross-sectional area. Strain is defined as the change in length per unit length and was calculated by dividing observed displacement with the original sample length. As mentioned earlier, the strain rate defined as the percentage change in strain per unit time was kept constant. Figure 2 shows the diagrammatic representation of stress, strain and strain rate definitions. Further, Young's modulus was calculated as the slope of linear region observed in the stress-strain curve. This was measured by providing a linear fit in the observed linear region of the curve and the slope value was extracted from the equation of linear fit. Figure 3 shows force vs. displacement curve and derived stress-strain curve and Young's modulus for testing of one of the raw potato sample. The stress-strain curve analysis and estimation of Young's modulus were done for each tested sample and each measurement was repeated in triplicates. The average value of the estimated Young's moduli was considered for analysis. Also, after every instant of compression testing, the deformed sample was removed and the compression platen probe and sample platform was cleaned well with acetone and the probe was lubricated to avoid the friction between its surface and food sample.

\section{Results and discussion}

The effect of change in sample's shape, geometry, moisture content and applied strain rate on Young's modulus and compressive strength estimates was investigated. We demonstrate that although variations of the same order in size, aspect ratio or shape may not significantly affect Young's modulus and compressive strength estimates of food materials, the strain rate has a statistically significant effect on these properties, thus exhibiting the viscoelastic behaviour. The viscoelastic behaviour was further investigated by varying test procedures and food type. Finally, the effect of thermal processes and associated moisture loss on the variation in Young's modulus was analysed.

Figure 4 shows Young's modulus and compressive strength during compression testing of potato samples of different geometries, aspect ratio and dimensions. Table 1 describes the various cases considered. Comparison of test case 1, 2 and 3 shows that there is no significant effect of aspect ratio on the compressive strength and Young's modulus. Comparison with case 4 indicates that geometry does not affect the results significantly (statistical analysis is done by performing one way ANOVA (Analysis of Variance) for a single factor using Analysis Toolpak embedded in Microsoft Excel Professional Plus 2013) with a confidence interval of $95 \%(\alpha=0.05)$. In both cases, the obtained $p$-value was greater than $\alpha$, and therefore we may conclude that there is no effect of geometry and aspect ratio on Young's modulus and compressive strength obtained. Analyses results are shown in Tables 2 and 3 respectively.

Next, the effect of strain rate on Young's modulus and compressive strength was investigated (Figure 5). A similar analysis (as was carried out for the effect of shape and aspect ratio) yielded a statistically significant effect of strain rate on the estimated Young's modulus (up to 54\% variation) and compressive strength values (up to $29 \%$ variation) (Tables 4 and 5). This indicates the strong need for standardization of test procedures that would reduce variation in the reported values of Young's modulus and compressive strength across the literature.

The dependence of Young's modulus on the strain rate also indicates viscoelastic behaviour of materials [41]. In order to further validate this behaviour, stress-relaxation tests and cyclic tests were carried out. Procedure for conducting these tests are referred from the literature [42], 


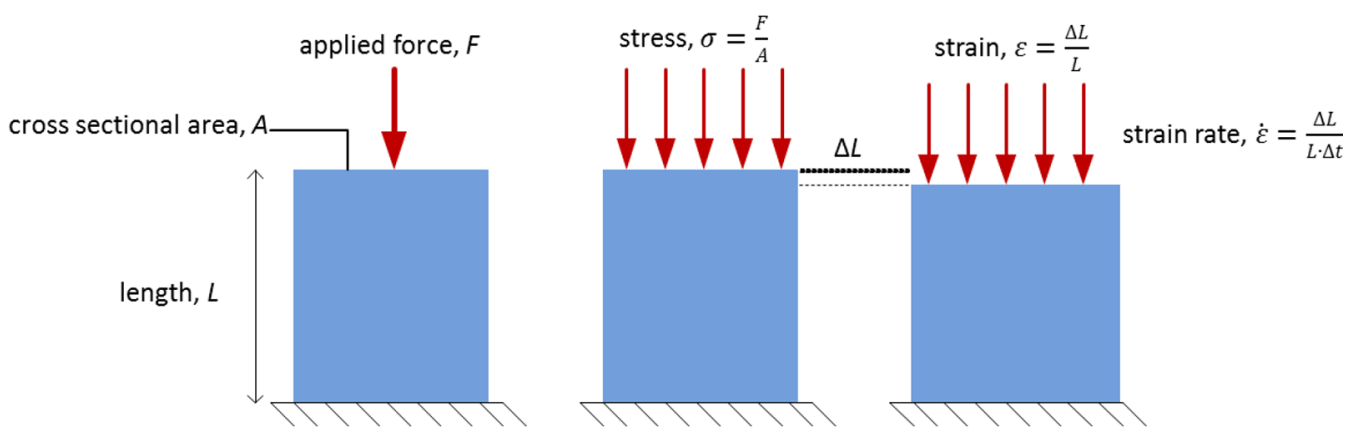

Fig. 2. Stress, strain and strain rate definition.
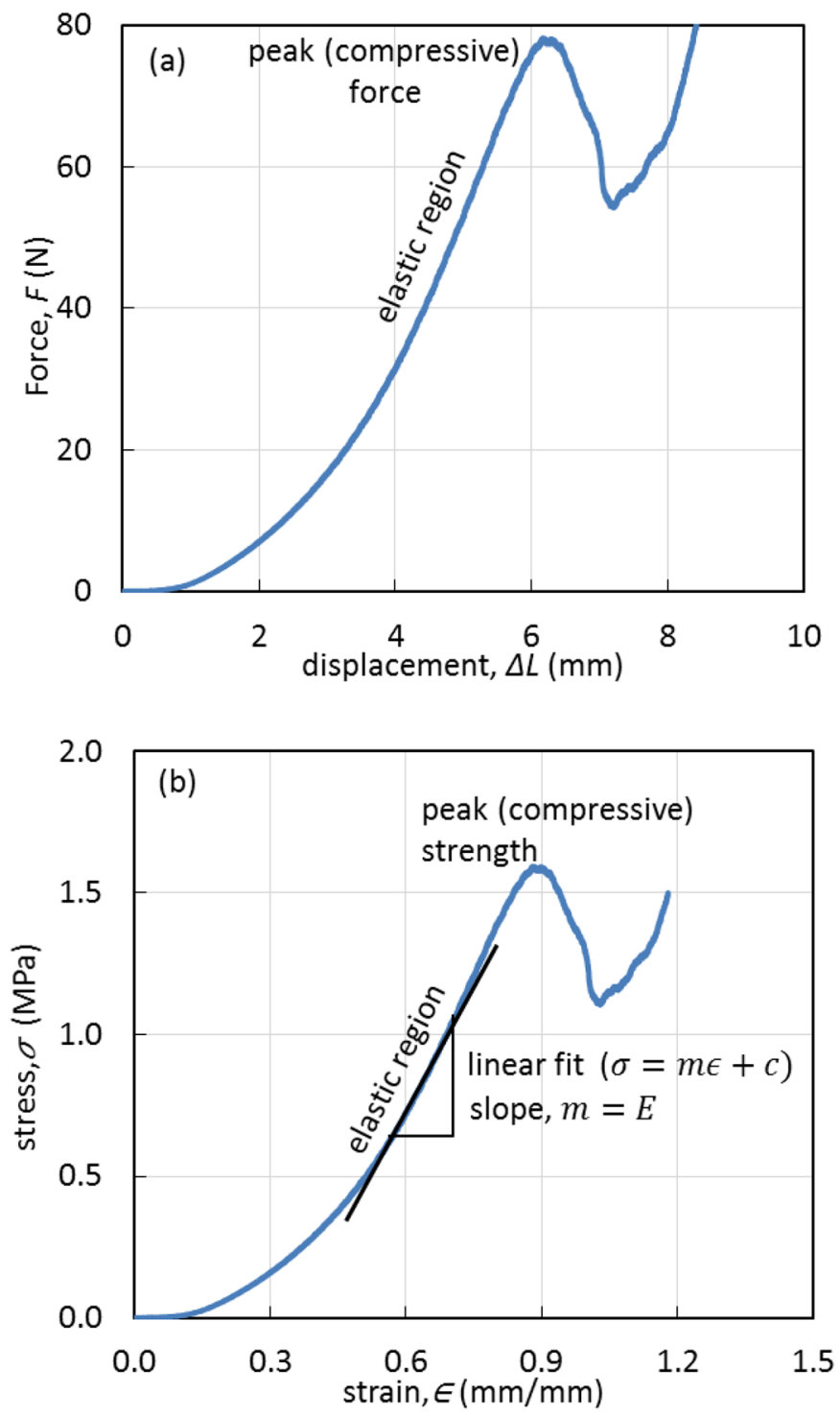

Fig. 3. (a) Force vs. displacement curve obtained from texture analysis and (b) derived stress-strain curve.

whereas sample dimensions were chosen as mentioned earlier. Figure 6 shows the observed stress relaxation behaviour and effect of applied strain rate on it. Unlike ideal elastic behaviour, a decrease in residual stress was

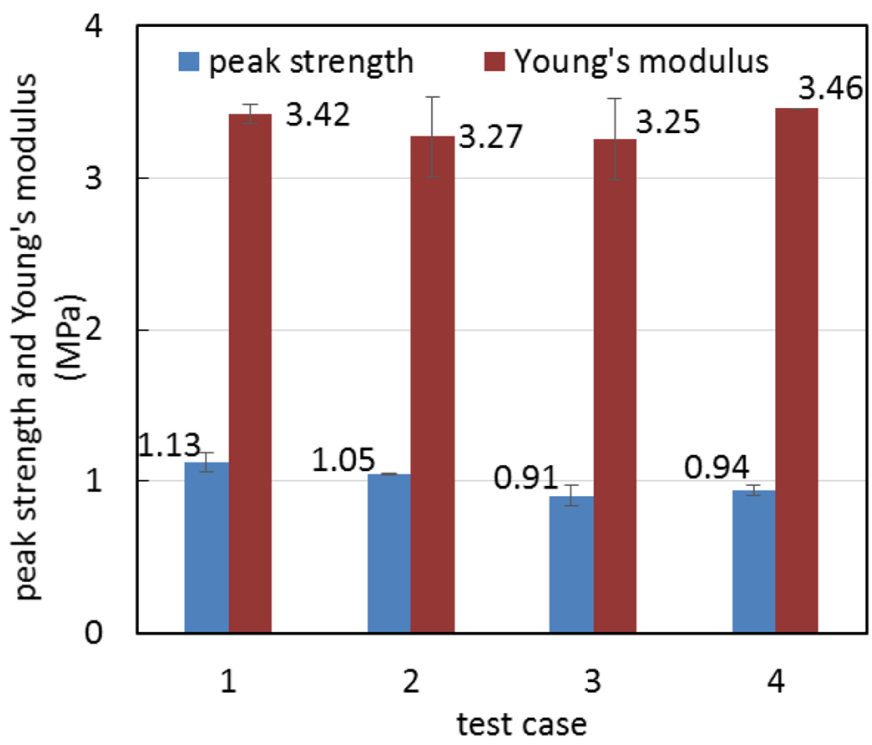

Fig. 4. Geometry and aspect ratio do not significantly affect values of Young's modulus and compressive strength (see also Table 1).

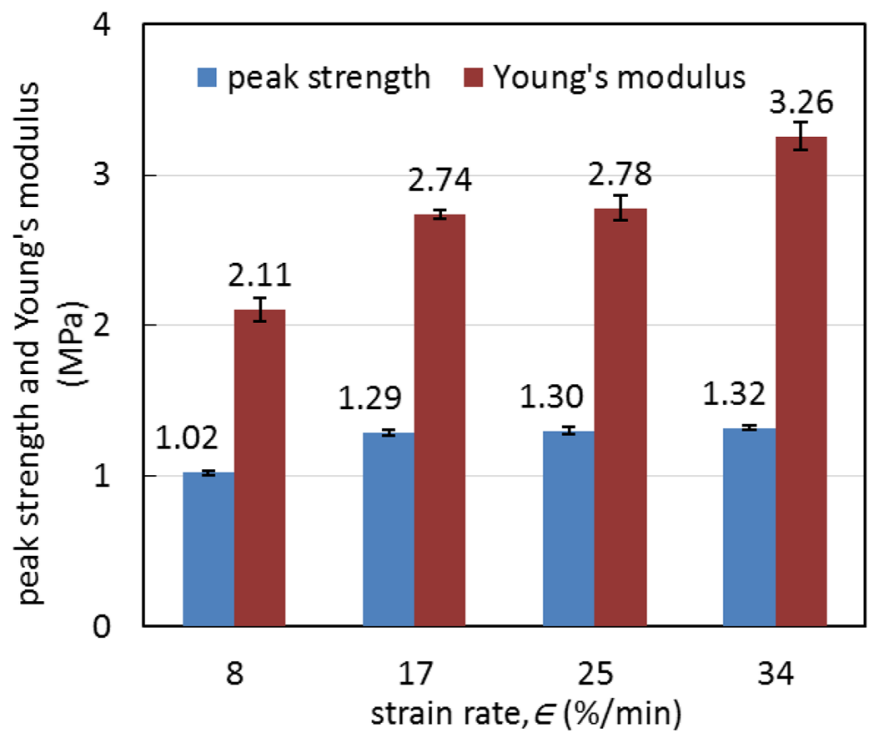

Fig. 5. Strain rate affects estimates of Young's modulus and compressive strength, indicating the need for standardization of tests for food materials. 
Table 2. Analysis of variance (ANOVA) shows that aspect ratio or geometry do not (statistically) affect values of Young's modulus.

\begin{tabular}{lllllll}
\hline ANOVA & & & & & \\
Source of variation & $\mathrm{SS}$ & $\mathrm{df}$ & $\mathrm{MS}$ & $F$ & 0.867 & 4.066 \\
\hline Between groups & 0.093 & 3 & 0.031 & 0.239 & & \\
Within groups & 1.031 & 8 & 0.129 & & & \\
Total & 1.123 & 11 & & & & \\
\hline
\end{tabular}

Table 3. ANOVA shows that aspect ratio or geometry do not (statistically) affect values of compressive strength.

\begin{tabular}{lllllll}
\hline ANOVA & & & & & \\
Source of variation & $\mathrm{SS}$ & $\mathrm{df}$ & $\mathrm{MS}$ & $F$ & $p$-value & $F_{\text {critical }}$ \\
\hline Between groups & 0.112 & 3 & 0.037 & 2.828 & 0.107 & 4.066 \\
Within groups & 0.106 & 8 & 0.013 & & & \\
Total & 0.218 & 11 & & & & \\
\hline
\end{tabular}

Table 4. Young's modulus is affected by the strain rate.

\begin{tabular}{lllllll}
\hline ANOVA & & & & & \\
Source of variation & $\mathrm{SS}$ & $\mathrm{df}$ & $\mathrm{MS}$ & $F$ & $p$-value & $F_{\text {critical }}$ \\
\hline Between groups & 3.651 & 3 & 1.217 & 43.763 & $1.79 \times 10^{-8}$ & 3.160 \\
Within groups & 0.501 & 18 & 0.028 & & & \\
Total & 4.151 & 21 & & & & \\
\hline
\end{tabular}

Table 5. Peak strength is affected by strain rate.

\begin{tabular}{lllllll}
\hline $\begin{array}{l}\text { ANOVA } \\
\text { Source of variation }\end{array}$ & SS & df & MS & $F$ & $p$-value & $F_{\text {critical }}$ \\
\hline Between groups & 0.339 & 3 & 0.113 & 56.426 & $2.36 \times 10^{-9}$ & 3.160 \\
Within groups & 0.036 & 18 & 0.002 & & & \\
Total & 0.375 & 21 & & & & \\
\hline
\end{tabular}

observed with passage of time. This is due to the stress relaxation arising because of the delay in rearrangement of the internal structure of the sample. Some of the possible mechanisms of rearrangements and stress relaxation are molecular rearrangements and relaxation due to fluid flow and can be referred to the literature [41]. Figure 7 shows the hysteresis loop observed during cyclic loading and unloading tests of the potato sample. The hysteresis exhibits the partial recovery of energy at unloading. This is due to the phase lag between strain and stress arising due to delay in structure rearrangement [41].

In order to demonstrate that viscoelastic behaviour is not specific to potato samples only, tests were also performed for sweet potatoes. Figure 8 shows the stress relaxation behaviour and hysteresis losses during cyclic tests for sweet potatoes. Further, a comparison of stress relaxation and hysteresis behaviour of potato and sweet potato for the same sample dimensions and strain rate is shown in Figure 9.

Figures 10 and 11 show the results for variation in Young's modulus with change in moisture content for potato samples for drying and deep-frying cases, respectively. With decrease in moisture content, Young's modulus of potato samples increases during drying process. This behaviour is in agreement with other reported work on the dependence of Young's modulus with moisture content $[35,43]$. For deep frying processes, the behaviour is similar except in the initial region where a decrease in Young's modulus is observed. This is mainly because of the initial softening that occurs due to dominance of starch gelatinization [44-48].

Although the variation in Young's modulus with moisture content for different food materials have been reported by various researchers, theoretical explanation of 


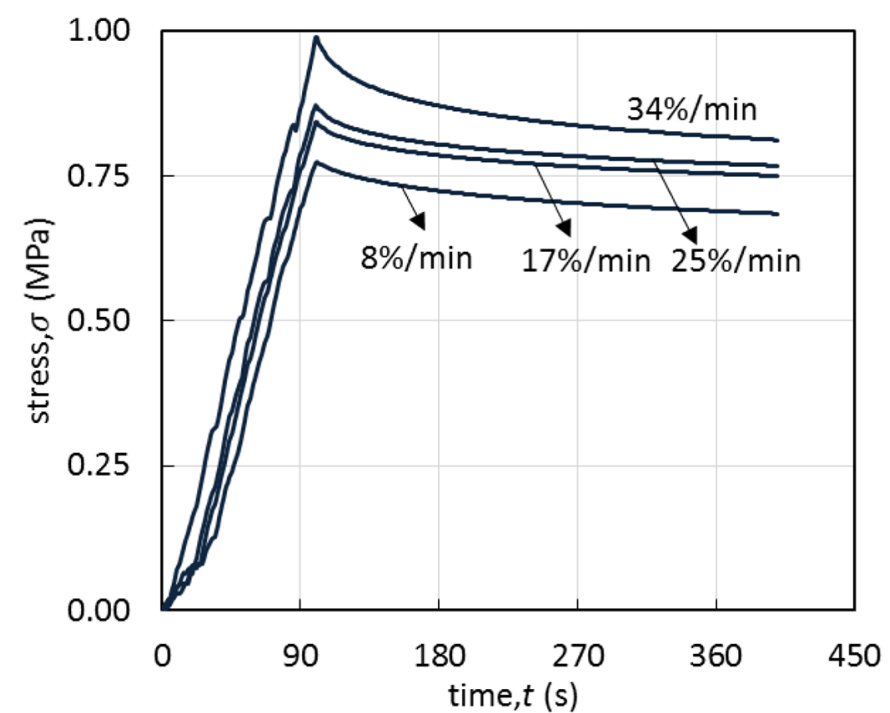

Fig. 6. Observed stress relaxation behaviour in raw potato samples indicates its viscoelastic nature, and strain rate affects this behaviour.

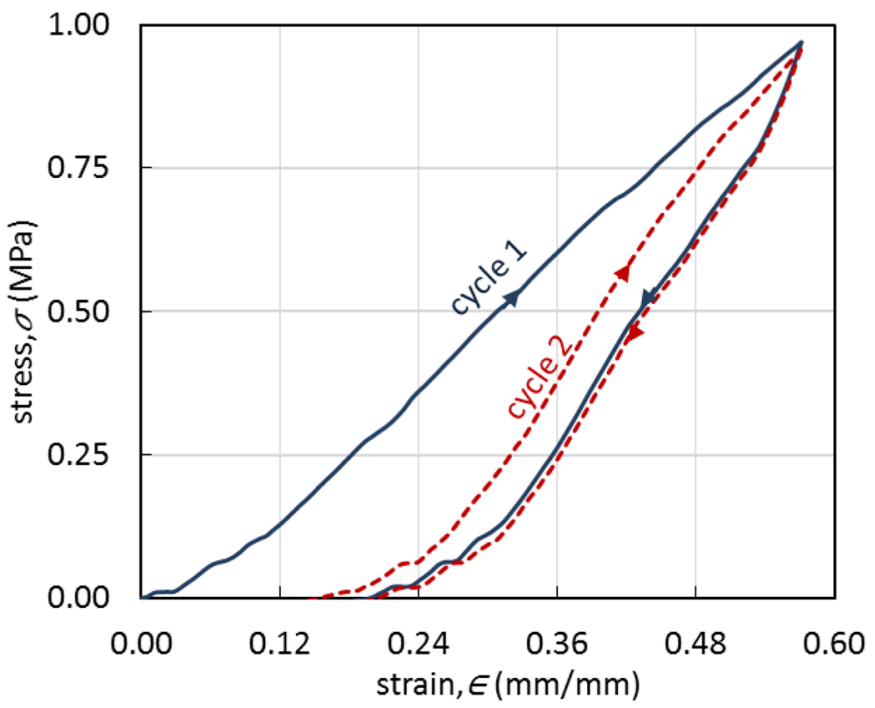

Fig. 7. Cyclic loading and unloading tests for raw potato samples: hysteresis loop during cyclic loading and unloading of the sample shows the energy loss due to phase lag between stress and strain while unloading. This observed behaviour confirms viscoelastic nature of the potato.

this behaviour has not been explained so far. The effect of moisture of Young's modulus could be explained on the basis of Terzhagi theory of effective stress [49]. Though the theory was originally proposed with reference to soil mechanics, it is applicable to other porous structures such as food materials as well. According to this theory, the net effective stress offered by the food material will be the difference of applied force per unit area and the pore water pressure. With a decrease in moisture content, pore water pressure decreases resulting in an increase in net effective stress which in turn results in increased strength and stiffness of material.
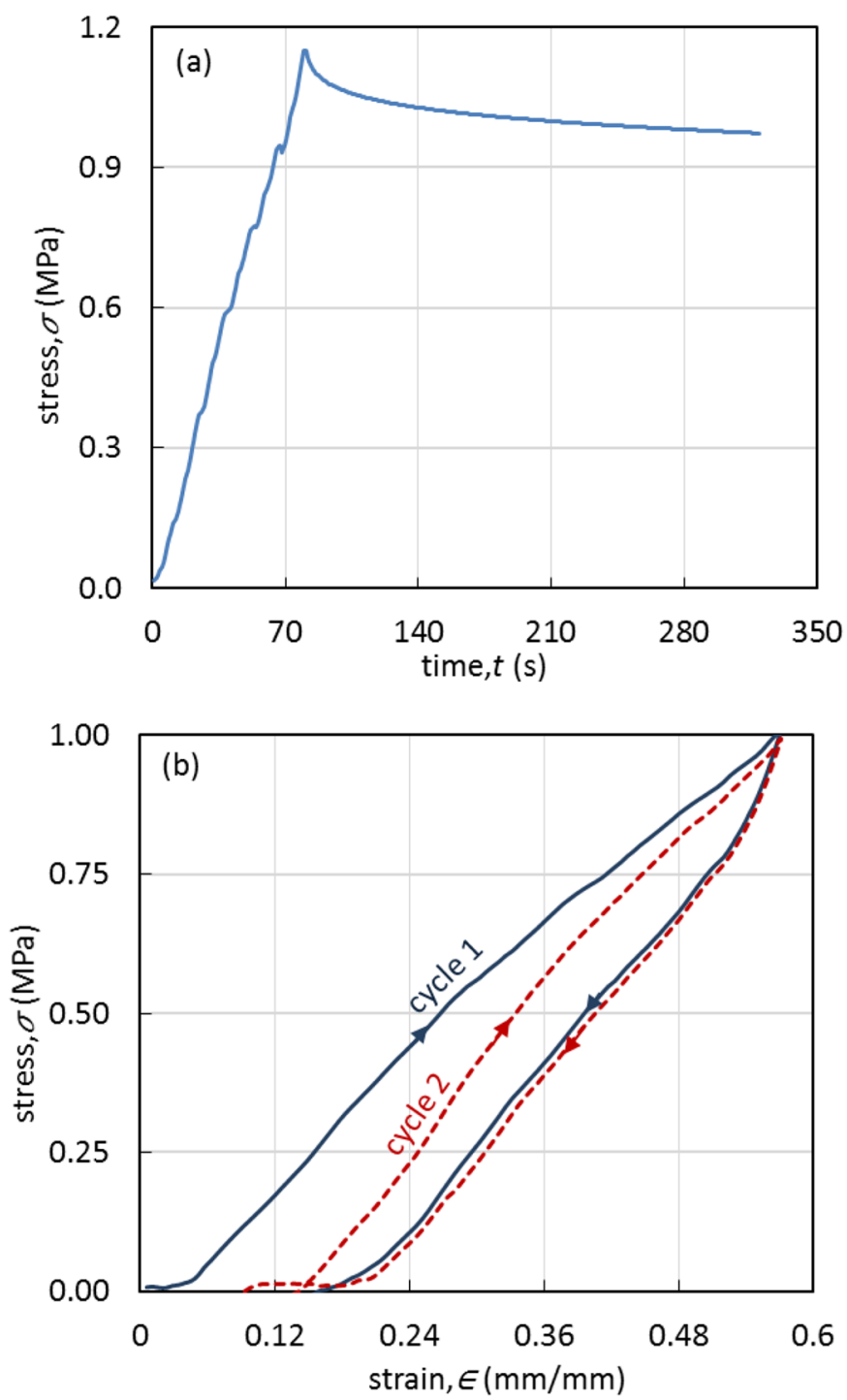

Fig. 8. Investigation of viscoelastic behaviour in raw sweet potato samples (a) stress relaxation behaviour (b) behaviour during cyclic tests.

The observed dependence of Young's modulus on moisture highlights the possibility of its variation even for the same samples procured from different geography or harvesting procedure since these can lead to different initial moisture content. Thus, development of standardised protocol for Young's modulus measurement also demands to indicate measured initial Young's Modulus along with its moisture content. Consequently, for measuring Young's modulus during thermal processes; the measurements should be considered as a function of moisture content as well, along with time. This will ensure better comparisons among the reported values by various researchers.

Also, it is important to note that the uniaxial compression of a sample may lead to sample expansion in directions perpendicular to the compression. This is an important phenomenon and is quantified by Poisson's ratio, defined as the ratio of negative of transverse strain to axial strain. It has been reported by various researchers 

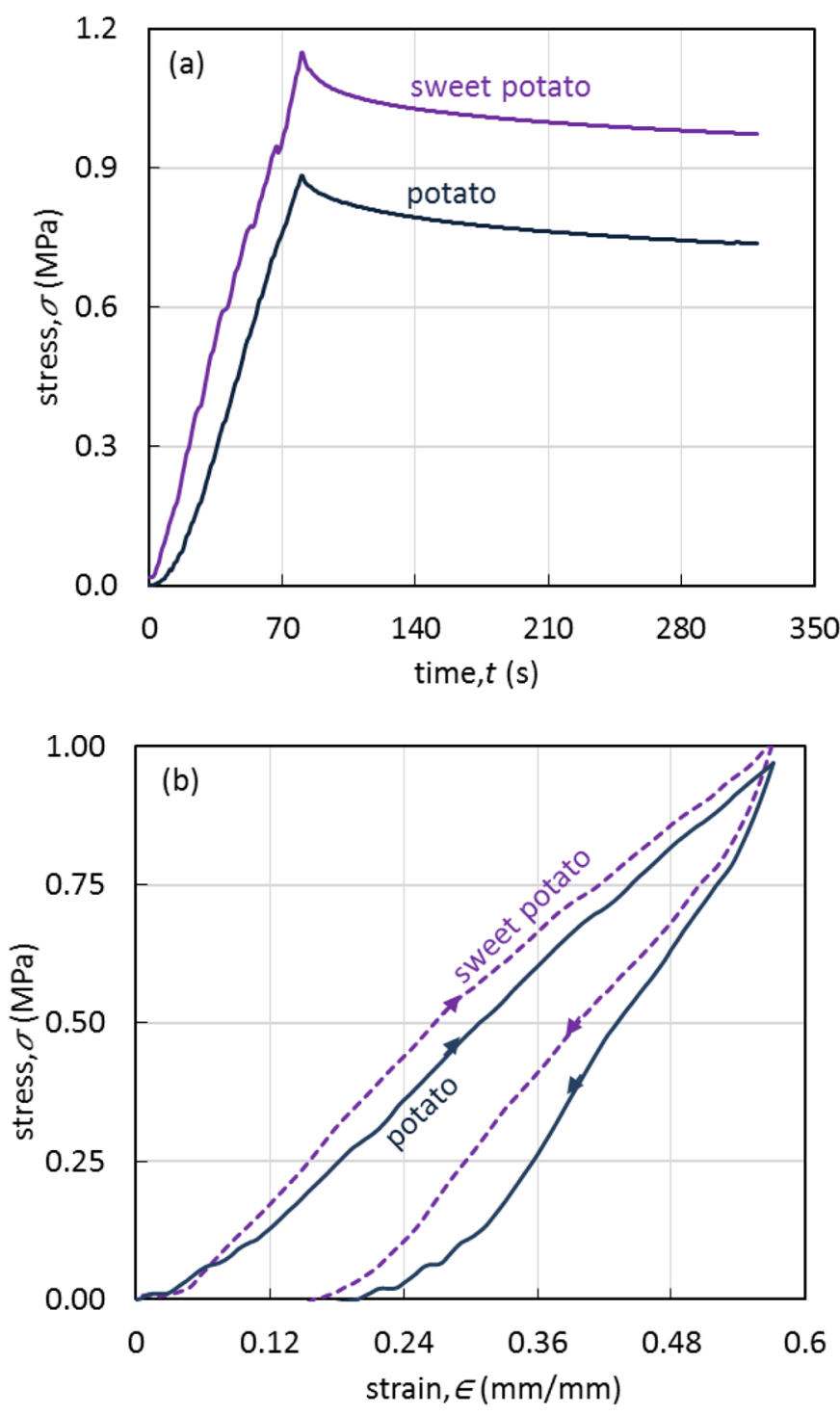

Fig. 9. Comparison of viscoelastic behaviour in potato vs. sweet potato. (a) stress relaxation behaviour (b) behaviour during cyclic tests.

that soft food materials such as potato comprise of parenchymatous tissues (with rubbery nature) and Poisson's ratio has been experimentally estimated to be between 0.49 and 0.5 [42,50-52]. However, Poisson's ratio deviates significantly below glass temperature as the material loses its rubbery behaviour and rigidity increases. The value of Poisson's ratio in glassy state varies from 0.25 to 0.33 [53]. A value of 0.3 has been suggested for potatoes in glassy state [50]. The information of Young's along with Poisson's ratio suffices for describing the deformation behaviour of a material [28]. Thus, Young's modulus estimation is the only crucial step for analysing deformation behaviour of such food materials.

Based on findings from the current work, we propose the following considerations for developing a standardised protocol for Young's modulus measurement:

- Strain rate should be held constant across the set of experiments.

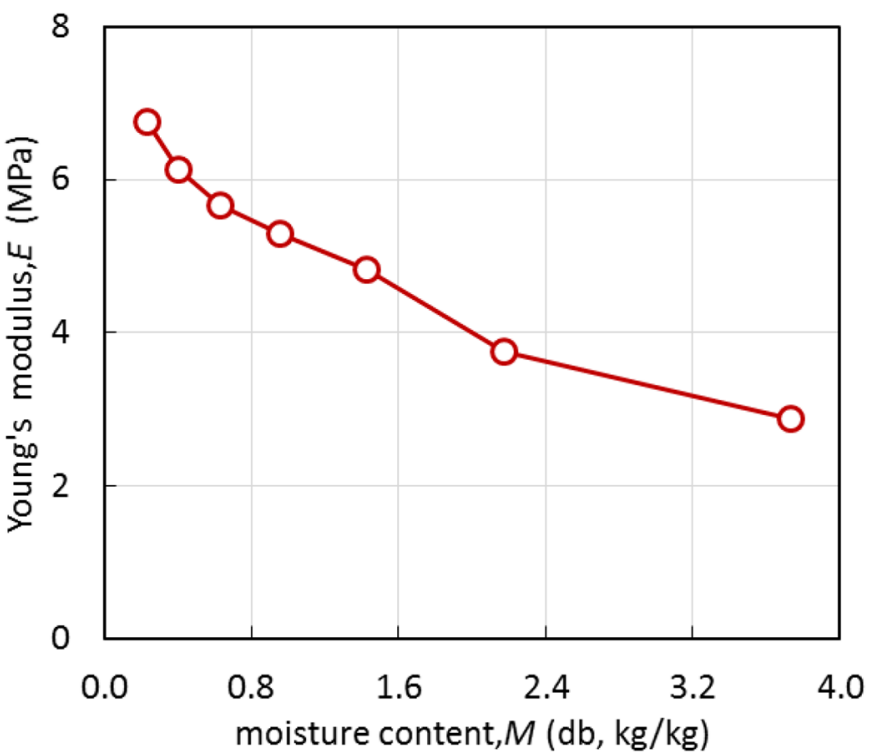

Fig. 10. Young's modulus increases with decreasing moisture content during drying.

- Information about the corresponding moisture content value should be mentioned with the obtained Young's modulus value.

- Samples with high aspect ratio may bulge or buckle during experiments and therefore should be avoided.

- For food materials with large lateral deformation, information about Poisson's ratio must also be supplemented along with Young's modulus.

\section{Conclusions}

In this paper, Young's modulus is proposed as a suitable measure of texture over other texture parameters due to its intensive nature and independence with respect to sample geometry and dimensions. Experimental stress-strain curves are analysed for different measurement conditions, and it is found that strain rate affects Young's modulus values by as much as $54 \%$, and compressive strength values by as much as $29 \%$. Therefore, need for standardisation of texture measurement procedures is established through experiments conducted in this paper. Further, the effect of moisture content on Young's modulus variation and its deviation due to starch gelatinization is discussed. The development of standard techniques of texture measurement can be utilized in future for analysing the effect of various other parameters such as gelatinization, temperature, other heat treatment processes on food texture.

\section{Funding}

Ankita Sinha receives stipend support from the Ministry of Human Resource Development (MHRD), Govt. of India. This research did not receive any specific grant from funding agencies in the commercial, public or not-for-profit sectors. 

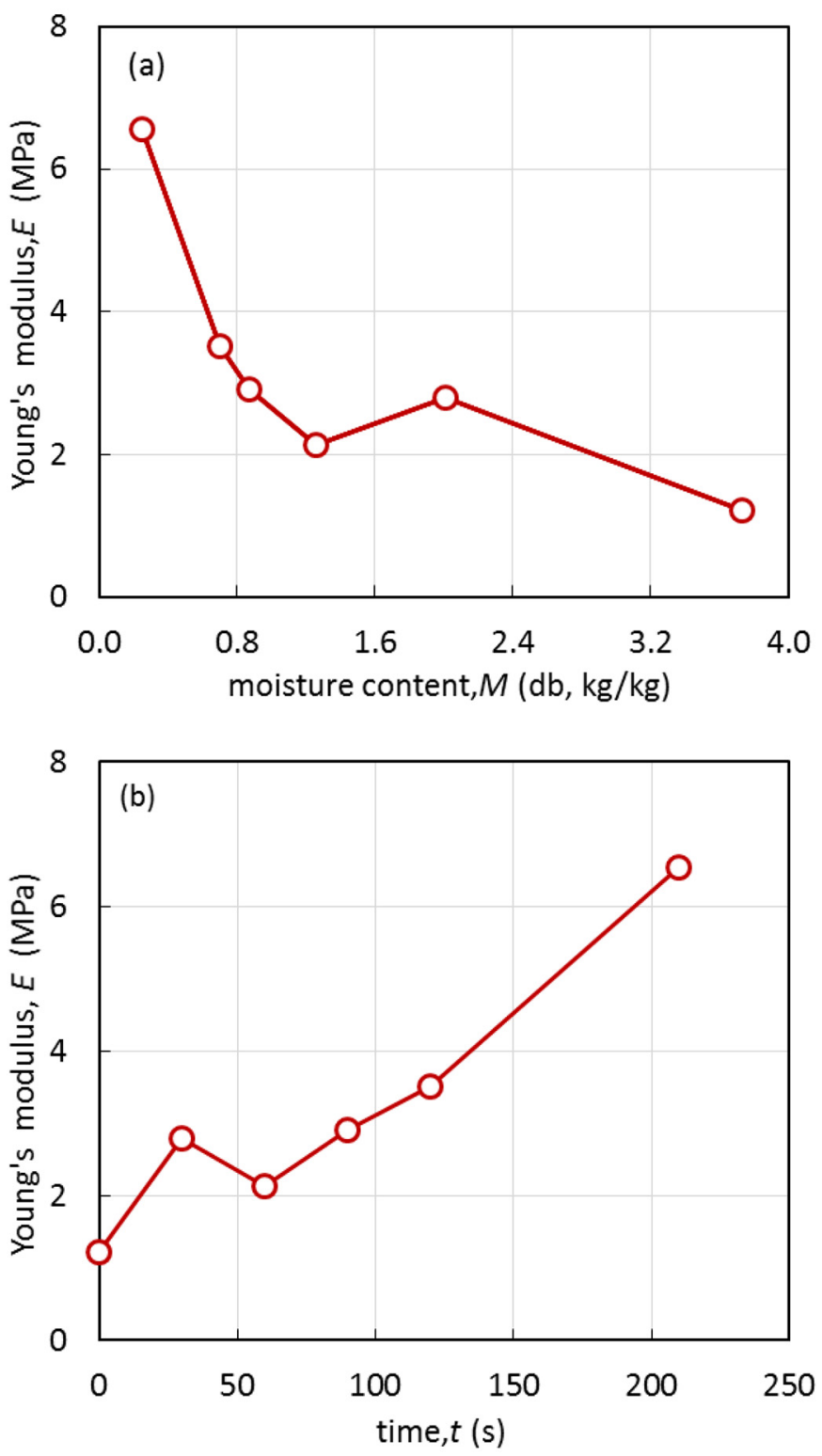

Fig. 11. Variation in Young's modulus with (a) moisture content and (b) time during deep-frying: Initial softening is observed during the onset of process followed by increase in Young's modulus with time.

The authors would like to acknowledge the support provided by Mr Shashank Shekhar (PhD student, IIT Gandhinagar) in instrument handling and operations for stress relaxation experiments.

\section{References}

[1] W.Y. Akwetey, C.L. Knipe, Sensory attributes and texture profile of beef burgers with gari, Meat Sci. 92, 745-748 (2012)

[2] L. Chen, U.L. Opara, Approaches to analysis and modeling texture in fresh and processed foods - a review, J. Food Eng. 119, 497-507 (2013)
[3] F. Costa et al., Assessment of apple (Malus $\times$ domestica Borkh.) fruit texture by a combined acoustic-mechanical profiling strategy, Postharvest Biol. Technol. 61, 21-28 (2011)

[4] E.H.J.H.-J. KIM, V.K. Corrigan, A.J. Wilson, I.R. Waters, D.I. Hedderley, M.P. Morgenstern, Fundamental fracture properties associated with sensory hardness of brittle solid foods, J. Texture Stud. 43, 49-62 (2012)

[5] D. Konopacka, W.J. Plocharski, Effect of storage conditions on the relationship between apple firmness and texture acceptability, Postharvest Biol. Technol. 32, 205-211 (2004)

[6] V. Stejskal et al., Sensory and textural attributes and fatty acid profiles of fillets of extensively and intensively farmed Eurasian perch (Perca fluviatilis L.), Food Chem. 129, 1054-1059 (2011)

[7] M. Taniwaki, K. Kohyama, Mechanical and acoustic evaluation of potato chip crispness using a versatile texture analyzer, J. Food Eng. 112, 268-273 (2012)

[8] R. Wang, W. Zhou, M. Isabelle, Comparison study of the effect of green tea extract (GTE) on the quality of bread by instrumental analysis and sensory evaluation, Food Res. Int. 40, 470-479 (2007)

[9] L. Chaunier, G. Della Valle, D. Lourdin, Relationships between texture, mechanical properties and structure of cornflakes, Food Res. Int. 40, 493-503 (2007)

[10] A. De Roeck, J. Mols, T. Duvetter, A. Van Loey, M. Hendrickx, Carrot texture degradation kinetics and pectin changes during thermal versus high-pressure/hightemperature processing: a comparative study, Food Chem. 120, 1104-1112 (2010)

[11] S. Farris, S. Gobbi, D. Torreggiani, L. Piergiovanni, Assessment of two different rapid compression tests for the evaluation of texture differences in osmo-air-dried apple rings, J. Food Eng. 88, 484-491 (2008)

[12] P. Greve, Y.S. Lee, J.F. Meullenet, B. Kunz, Improving the prediction for sensory texture attributes for multicomponent snack bars by optimizing instrumental test conditions, J. Texture Stud. 41, 358-380 (2010)

[13] L. Ragni, A. Berardinelli, A. Guarnieri, Impact device for measuring the flesh firmness of kiwifruits, J. Food Eng. 96, 591-597 (2010)

[14] V.B. Sasikala, R. Ravi, H.V. Narasimha, Textural changes of green gram (Phaseolus aureus) and horse gram (Dolichos biflorus) as affected by soaking and cooking, J. Texture Stud. 42, 10-19 (2011)

[15] D.N. Sila, C. Smout, F. Elliot, A. Van Loey, M. Hendrickx, Non-enzymatic depolymerization of carrot pectin: toward a better understanding of carrot texture during thermal processing, J. Food Sci. 71, E1-E9 (2006)

[16] A.P. Cherng, F. Ouyang, A firmness index for fruits of ellipsoidal shape, Biosyst. Eng. 86, 35 (2003)

[17] I. Shmulevich, N. Galili, M.S. Howarth, Nondestructive dynamic testing of apples for firmness evaluation, Postharvest Biol. Technol. 29, 287-299 (2003)

[18] M. Taniwaki, T. Hanada, N. Sakurai, Postharvest quality evaluation of 'Fuyu' and 'Taishuu' persimmons using a nondestructive vibrational method and an acoustic vibration technique, Postharvest Biol. Technol. 51, 80-85 (2009)

[19] L.T. Nguyen, A. Tay, V.M. Balasubramaniam, J.D. Legan, E.J. Turek, R. Gupta, Evaluating the impact of thermal and pressure treatment in preserving textural quality of selected foods, LWT-Food Sci. Technol. 43, 525-534 (2010) 
[20] S. Ichiro Iwatani, H. Yakushiji, N. Mitani, N. Sakurai, Evaluation of grape flesh texture by an acoustic vibration method, Postharvest Biol. Technol. 62, 305-309 (2011)

[21] M. Taniwaki, T. Hanada, N. Sakurai, Device for acoustic measurement of food texture using a piezoelectric sensor, Food Res. Int. 39, 1099-1105 (2006)

[22] M. Taniwaki, N. Sakurai, Texture measurement of cabbages using an acoustical vibration method, Postharvest Biol. Technol. 50, 176-181 (2008)

[23] B.V. Pamies, G. Roudaut, C. Dacremont, M. Le Meste, J.R. Mitchell, Understanding the texture of low moisture cereal products: mechanical and sensory measurements of crispness, J. Sci. Food Agric. 80, 1679-1685 (2000)

[24] M. Kristiawan, L. Chaunier, G. Della Valle, D. Lourdin, S. Guessasma, Linear viscoelastic properties of extruded amorphous potato starch as a function of temperature and moisture content, Rheol. Acta 55, 597-611 (2016)

[25] Z. Liu, M.G. Scanlon, Predicting mechanical properties of bread crumb, Food Bioprod. Process. 81, 224-238 (2003)

[26] R. Kadowaki, H. Kimura, N. Inou, New estimation methods of Young's modulus and rupture strength of snack foods based on microstructure, J. Texture Stud. 47, 3-13 (2016)

[27] S. Thussu, A.K. Datta, Texture prediction during deep frying: a mechanistic approach, J. Food Eng. 108, 111-121 (2012)

[28] E.P. Popov, T.A. Balan, Engineering mechanics of solids, vol. 2. Prentice Hall Englewood Cliffs, NJ (1990)

[29] L. Mioche, M.A. Peyron, Bite force displayed during assessment of hardness in various texture contexts, Arch. Oral Biol. 40, 415-423 (1995)

[30] K.R. Agrawal, P.W. Lucas, I.C. Bruce, J.F. Prinz, Food properties that influence neuromuscular activity during human mastication, J. Dent. Res. 77, 1931-1938 (1998)

[31] K.R. Agrawal, P.W. Lucas, J.F. Prinz, I.C. Bruce, Mechanical properties of foods responsible for resisting food breakdown in the human mouth, Arch. Oral Biol. 42, 1-9 (1997)

[32] S.H. Williams, B.W. Wright, V. den Truong, C.R. Daubert, C.J. Vinyard, Mechanical properties of foods used in experimental studies of primate masticatory function, Am. J. Primatol. 67, 329-346 (2005)

[33] T. Takeshita, F. Nakazawa, Mastication velocity of the first molar in relation to the mechanical properties of food, J. Home Econ. Jpn. 58, 129 (2007)

[34] H. Dan, K. Kohyama, Interactive relationship between the mechanical properties of food and the human response during the first bite, Arch. Oral Biol. 52, 455-464 (2007)

[35] M. Kiani Deh Kiani, H. Maghsoudi, S. Minaei, Determination of poisson's ratio and young's modulus of red bean grains, J. Food Process Eng. 34, 1573-1583 (2011)
[36] J.F.V. Vincent, Application of fracture mechanics to the texture of food, Eng. Fail. Anal. 11, 695-704 (2004)

[37] M.K. Krokida, V. Oreopoulou, Z.B. Maroulis, D. MarinosKouris, Effect of pre-treatment on viscoelastic behaviour of potato strips, J. Food Eng. 50, 11-17 (2001)

[38] M.K. Krokida, V. Oreopoulou, Z.B. Maroulis, D. MarinosKouris, Viscoelastic behaviour of potato strips during deep fat frying, J. Food Eng. 48, 213-218 (2001)

[39] M.C. Boyce, S. Socrate, P.G. Llana, Constitutive model for the finite deformation stress-strain behavior of poly (ethylene terephthalate) above the glass transition, Polyme 41, 2183-2201 (2000)

[40] J.W. Hutchinson, K.W. Neale, Influence of strain-rate sensitivity on necking under uniaxial tension, Acta Metall. 25, 839-846 (1977)

[41] S.R. Lakes, Viscoelastic solids. CRC Press (2018)

[42] T. van Vliet, Rheology and fracture mechanics of foods (2013)

[43] P. Mazumder, B.S. Roopa, S. Bhattacharya, Textural attributes of a model snack food at different moisture contents, J. Food Eng. 79, 511-516 (2007)

[44] M. Alvarez, W. Canet, M. Tortosa, Kinetics of thermal softening of potato tissue (cv. Monalisa) by water heating, Eur. Food Res. Technol. 212, 588-596 (2001)

[45] M.D. Alvarez, W. Canet, Kinetics of softening of potato tissue by temperature fluctuations in frozen storage, Eur. Food Res. Technol. 210, 273-279 (2000)

[46] A. Andersson, V. Gekas, I. Lind, F. Oliveira, R. Öste, J.M. Aguilfra, Effect of preheating on potato texture, Crit. Rev. Food Sci. Nutr. 34, 229-251 (1994)

[47] Y.T. Huang, M.C. Bourne, Kinetics of thermal softening of vegetables, J. Texture Stud. 14, 1-9 (1983)

[48] A.R. Taherian, Thermal softening kinetics and textural quality of thermally processed vegetables, MSc Thesis, Dept. of Food Sc. and Agri. Chem., McGill Univ., Montreal, 1996. Available: https://escholarship.mcgill.ca/concern/the ses $/ 3$ f4627486? locale $=$ en

[49] K. Terzaghi, R.B. Peck, G. Mesri, Soil mechanics in engineering practice. John Wiley \& Sons (1996)

[50] T. Gulati, A.K. Datta, Mechanistic understanding of casehardening and texture development during drying of food materials, J. Food Eng. 166, 119-138 (2015)

[51] K.J. Niklas, Plant biomechanics: an engineering approach to plant form and function. University of Chicago Press (1992)

[52] E.E. Finney, C.W. Hall et al., Elastic properties of potatoes, Trans. ASAE 10, 4-8 (1967)

[53] V. Srivastava, S.A. Chester, N.M. Ames, L. Anand, A thermo-mechanically-coupled large-deformation theory for amorphous polymers in a temperature range which spans their glass transition, Int. J. Plast. 26, 1138-1182 (2010)

Cite this article as: A. Sinha, A. Bhargav, Young's modulus estimation in food samples: effect of experimental parameters, Mechanics \& Industry 21, 404 (2020) 